James C. Andrews, M.D., Neil A. Martin, M.D.,

Keith Black, M.D., Vincent F. Honrubia, M.D., and Donald P. Becker, M.D.

\title{
Middle Cranial Fossa
}

\section{Transtemporal Approach to the}

\section{Intrapetrous Internal Carotid Artery}

\begin{abstract}
Diseases involving the proximity of the internal carotid artery at the skull base require identification of this vessel in the temporal bone to gain vascular control for any maneuver in its vicinity. This article details the technique of surgical dissection and exposure of the internal carotid artery within the skull base through a transtemporal middle cranial fossa approach. The anatomic landmarks important in utilizing this procedure include the greater superficial petrosal nerve, the mandibular branch of the trigeminal nerve, and the middle meningeal artery. With this approach, the internal carotid artery can be followed throughout the carotid canal and beyond the foramen lacerum to the region of the cavernous sinus. The indications and limitations of this procedure are described and several clinical applications are provided.
\end{abstract}

Surgical procedures in the region of the internal carotid artery are challenging because of the morbidity or fatality that can result from injury to this vessel. In some patients sacrifice of the internal carotid can be a means to manage this artery when performed with proper monitoring in a controlled manner; however, in others this procedure can be disastrous. ${ }^{1,2}$ Operative maneuvers to locate, protect, and safely manipulate the internal carotid artery are necessary. This article details a direct approach to the intrapetrous internal carotid artery through the middle cranial fossa.

\section{MATERIALS AND METHODS}

Cadaver dissections provided the initial background for developing the middle cranial fossa transtemporal approach to the intrapetrous internal carotid artery. Clinical applications were based on the experience gained in the dissection laboratory. The intrapetrous internal carotid artery was approached through this procedure in six patients (Table 1). Five patients had a tumor of the skull base with invasion or intimate association of the distal internal carotid artery. One patient (case 2) had a vascular injury following closed head trauma. All patients were preoperatively studied with computed tomography (CT), magnetic resonance imaging (MRI), and angiography to delineate the extent of their condition and the involvement of the internal carotid artery.

\section{SURGICAL TECHNIQUE}

A wide C-shaped incision is utilized initially following the approximate origin of the temporalis muscle and continued into the preauricular region approximately to the level of the external auditory canal (Fig. 1). A plane of dissection is established over the temporalis fascia and a skin and subcutaneous tissue flap is elevated, encompassing the peripheral frontal branches of the facial nerve. The zygomatic arch is exposed and the superficial fascial attachments and periosteum are incised along its superior and lateral aspect to the region of the lateral orbit. The inferior attachments of the zygomatic arch, including the

Skull Base Surgery, Volume 1, Number 3, July 1991 Divisions of Head and Neck Surgery and Neurosurgery, University of California, Los Angeles School of Medicine, Los Angeles, California, and the Department of Otolaryngology, Northwestern University School of Medicine, Chicago, Illinois Reprint Requests: Dr. Andrews, Division of Head and Neck Surgery, 62-132 CHS, Center for the Health Sciences, Los Angeles, CA 90024-1624 
Table 1. Patients Requiring Middle Cranial Fossa Transtemporal Exposure of the Internal Carotid Artery

\begin{tabular}{ccl}
\hline Patient & Age & Pathology \\
\hline 1 & 56 & Sarcoma (recurrent) \\
2 & 45 & Traumatic intimal dissection of ICA \\
3 & 57 & Sarcoma \\
4 & 48 & Meningioma \\
5 & 49 & Glomus jugulare tumor (C4De1)* \\
6 & 29 & Chordoma \\
\hline
\end{tabular}

${ }^{*}$ C4De1 refers to the Fisch staging classification for glomus jugulare tumors. ${ }^{12}$

superficial fascia and the masseter muscle, are preserved. Using an oscillating saw, the zygomatic arch is divided at its root in the temporal bone and at the lateral aspect of the orbit. This allows the arch to be reflected inferiorly on a viable pedicle of the masseter muscle and superficial fascia. The temporalis muscle is incised at its origin in the temporal fossa of the skull and retracted inferiorly along its pedicle of insertion into the mandible.

A $3 \mathrm{~cm}^{2}$ low middle fossa craniotomy centered over the zygomatic root is prepared (Fig. 2). The inferior aspect of the craniotomy is created below the level of the zygomatic arch and continued through the inferior aspect of the squamous portion of the temporal bone and the lateral region of the greater wing of the sphenoid. The frontal branch of the middle meningeal artery is usually avoided by staying posterior to the region of its bony canal in the pterion. The inferior edge of the craniotomy is then lowered to the level of the middle cranial fossa floor.

The dura is elevated from the cranial floor to the

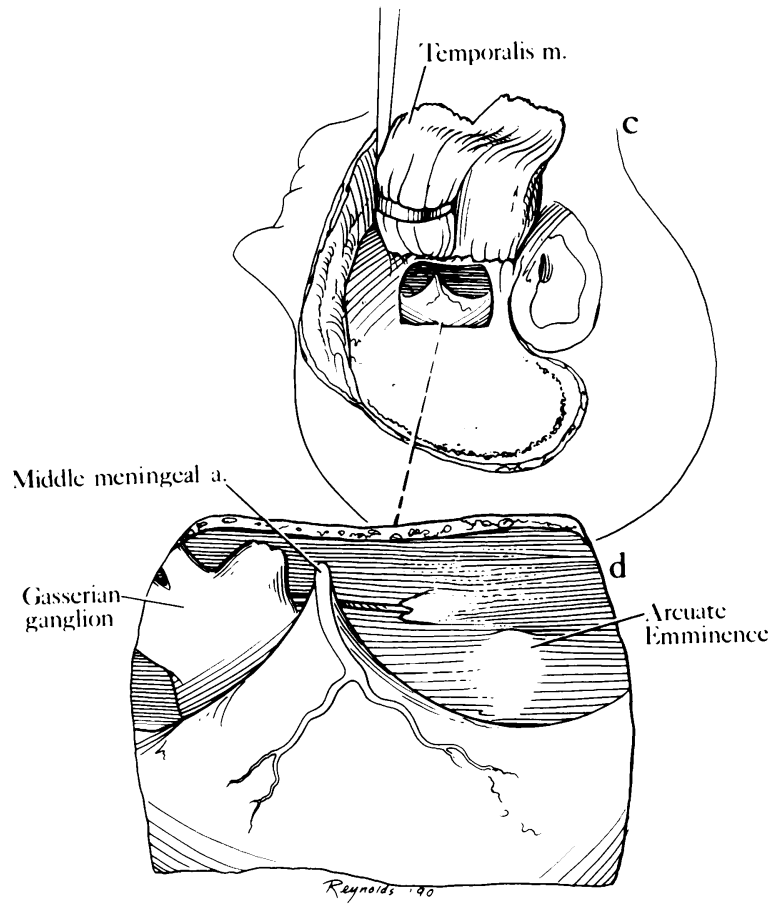

Figure 2. c: The temporalis muscle has been incised and elevated from its insertion into the temporal and parietal bones. A low middle cranial fossa craniotomy has been prepared. Insert (d) shows a detailed view of the middle cranial fossa craniotomy. The dura and brain fall away from the inferior edge of the craniotomy except for the attachments to the middle meningeal artery. Drilling will begin parallel and just medial to the greater superficial petrosal nerve.
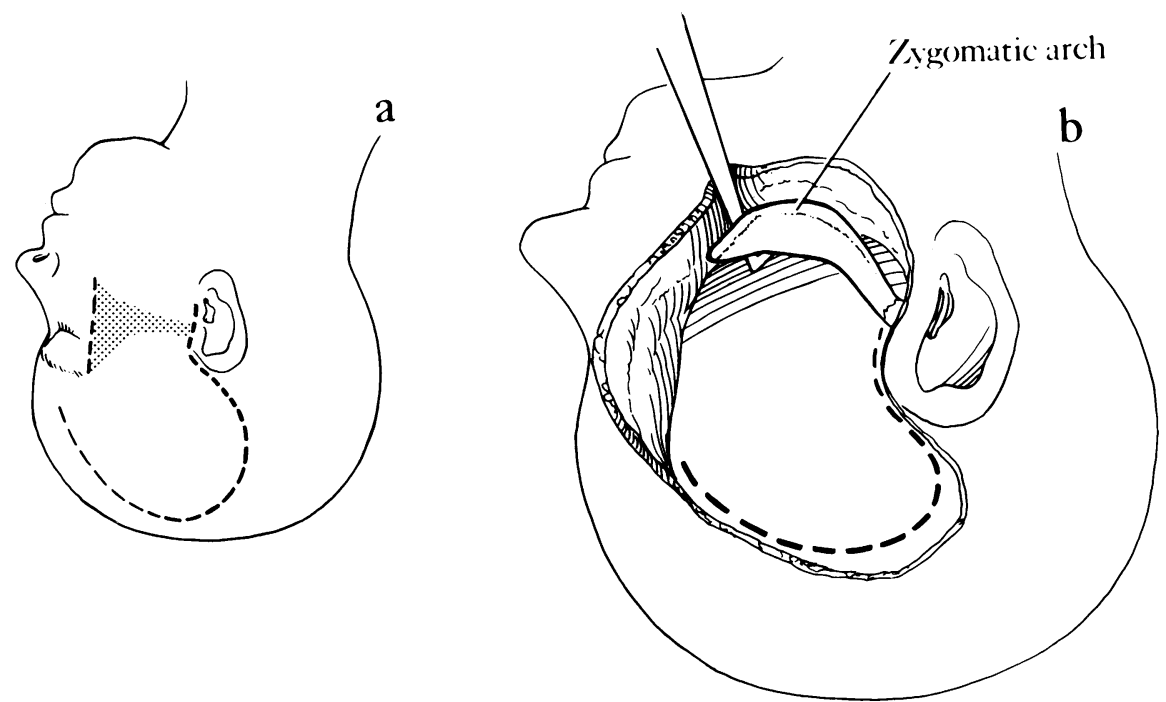

Figure 1. a: Skin incision proposed for the middle cranial fossa transtemporal approach to the intrapetrous internal carotid artery with orientation to the zygoma. b: A skin and subcutaneous tissue flap encompassing the peripheral branches of the facial nerve has been elevated. The zygomatic arch is being displaced on an inferior masseter muscle pedicle after having been divided at its root and its attachments to the orbital wall. 
region of the foramen spinosum. The middle meningeal artery is coagulated and divided. Further dural elevation is carried along the petrous ridge of the temporal bone. Key anatomic landmarks to be identified include the arcuate eminence, the greater superficial petrosal nerve, and the maxillary and mandibular branches of the trigeminal nerve as they, respectively, exit through the foramena rotundum and ovale. The horizontal course of the internal carotid artery lies deep in the petrous bone, parallel and medial to the greater superficial petrosal nerve from the region of the geniculate to the gasserian ganglion. The arcuate eminence provides approximate orientation to the labyrinth of the inner ear. Using a 2 to $3 \mathrm{~mm}$ diamond burr, the greater superficial petrosal nerve is uncovered proximally through the temporal bone from the facial hiatus back to the region of the geniculate ganglion. Drilling is then performed in the petrous ridge parallel and just medial to the greater superficial petrosal nerve in the region of the foramen spinosum to locate the internal carotid artery (Fig. 3). The eustachian tube may also be identified in the petrous bone lying lateral to the internal carotid. The internal carotid artery takes an oblique course through the petrous bone, being covered by a thin layer of bone near the trigeminal ganglion but lying much deeper near the labyrinth of the inner ear. After the internal carotid artery has been initially identified, further drilling can expand the exposure throughout the vessel's horizontal course and into the cavernous sinus region.

Reconstruction of the operative site includes reinsertion of the bone graft into the region of the craniotomy followed by replacement of the temporalis muscle back to its original position. The zygomatic arch is reapproximated and fixed in place using 20 gage wire.

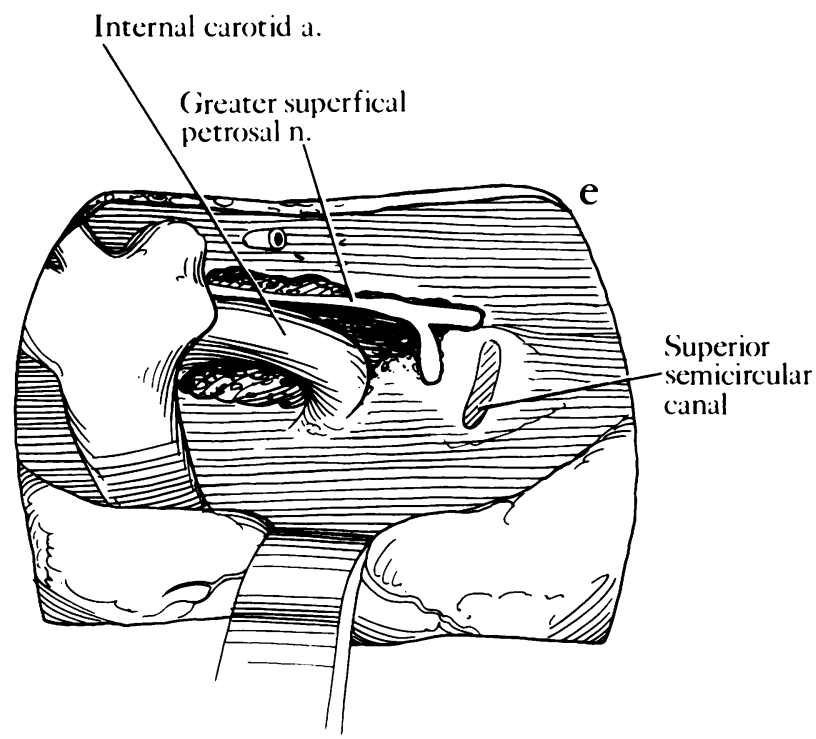

Figure 3. A detailed view of the exposed horizontal segment of the internal carotid artery.

\section{RESULTS}

Intrapetrous internal carotid artery exposure was performed without difficulty in all six cases of this study. In four of the five patients with tumors, the exposure facilitated identification of a plane of dissection around the internal carotid artery that enabled safe removal of the tumor. In one patient with a recurrent meningioma, injury to the internal carotid artery resulted from attempting to dissect between the adventitia of the vessel and the tumor. The exposure provided by this approach, however, allowed rapid control of hemorrhage and subsequent repair of the vessel. No other complications were encountered as a result of this procedure.

\section{ILLUSTRATIVE CASE REPORTS}

\section{Case 1}

A 68-year-old woman with unilateral facial pain was referred to UCLA Center for the Health Sciences for management of a sarcoma involving the superior clival region. CT, MRI, and angiographic evaluation showed the mass involved the foramen rotundum and extended along the internal carotid artery in the distal intrapetrous segment to the region of the cavernous sinus (Fig. 4). Middle cranial fossa transtemporal exploration allowed identification of the internal carotid artery proximal to the region of the lesion. A plane of dissection between the tumor capsule and the adventitia of the vessel enabled safe and complete removal of the tumor.

\section{Case 2}

A 42-year-old woman was evaluated 2 days after a motor vehicle accident with symptoms of recurrent transient right-sided motor deficits that would resolve over a period of hours. An arteriogram showed evidence of an intimal dissection of the internal carotid artery, which began in the region of the bifurcation of the common carotid artery and extended into the carotid canal within the petrous bone. Balloon occlusion of the involved internal carotid artery was not a possible therapeutic option because arteriographic studies, including a cross-compression test, demonstrated poor collateral blood flow through the circle of Willis. A proximal to distal internal carotid artery bypass procedure was planned. Cervical dissection allowed isolation of the internal carotid artery in the neck. Middle cranial fossa transtemporal exploration enabled exposure of the intrapetrous internal carotid artery (Fig. 5). Dense connective tissue encompassing the internal carotid artery at the foramen of the carotid canal had prevented distal propagation of the intimal dissection beyond this region. A saphenous vein graft replaced the 

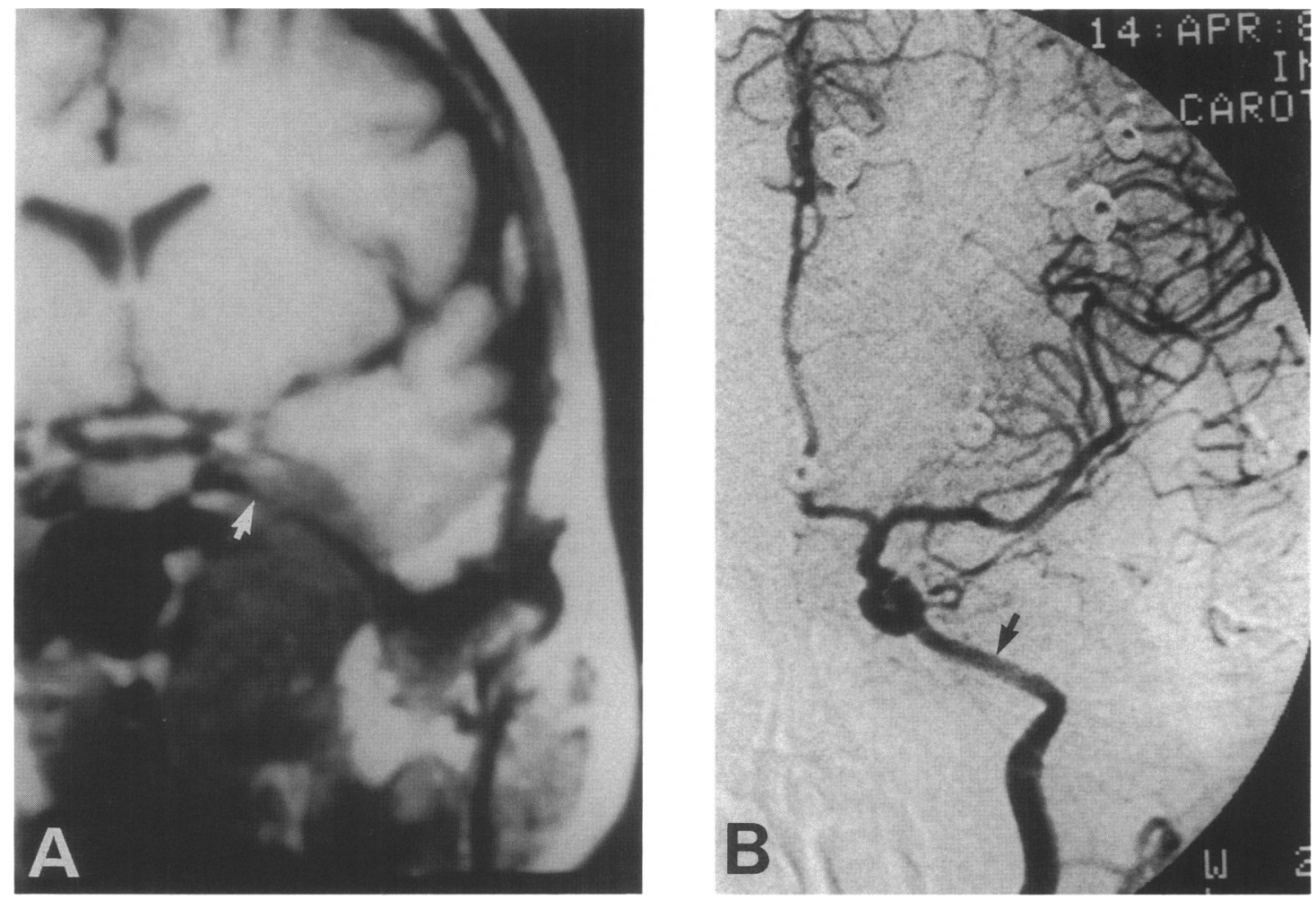

Figure 4. A: Coronal magnetic resonance imaging scan from the patient in case 1 demonstrating a mass extending to the horizontal region of the internal carotid artery (arrow). B: Arteriogram from the same patient showing the horizontal segment of the internal carotid artery surrounded by tumor (arrow).

diseased segment of vessel through two end-to-end anastomoses. A $1 \mathrm{~cm}^{2}$ segment of the craniotomy bone graft was removed to permit passage of the graft from the cervical region into the cranium.

\section{DISCUSSION}

Limited aspects of the middle cranial fossa transtemporal approach to the internal carotid artery have been described in various forms. ${ }^{3,4}$ Paullus et al ${ }^{5}$ provided anatomic dissections that detailed the landmarks and fundamental relationships in this region. Some of the clinical applications implicated for this procedure have included bypass operations for neoplastic infiltration of the vessel and vascular repairs for aneurysms and atheromatous lesions. ${ }^{6-8}$

The key advantage of the middle cranial fossa transtemporal approach is that it allows a direct means of intracranial extradural visualization of the intrapetrous internal carotid artery while maintaining the integrity of the ear. The exposure provided through this procedure is adequate to allow complex manipulation of the vessel, including dissection, ligation, repair, or vascular anastomoses.

Skull base neoplasms can result in significant distortion and displacement of the internal carotid artery as well as infiltration of the vessel wall. The carotid canal pro- vides a relatively protected site where the artery can be consistently identified. Since the carotid canal is often free of tumor involvement, this can also be an advantageous position to begin a plane of dissection between the adventitia of the artery and the tumor.

The limitations of this approach are few. The procedure requires extended drilling in the region of the greater superficial petrosal nerve and subsequently this structure is vulnerable to injury. The superior vertical segment of the internal carotid artery can only be minimally exposed through this approach. Other techniques, such as an infratemporal or transtemporal dissection, may provide better means to reach the high vertical segment of this vessel.9-11

\section{SUMMARY}

The middle cranial fossa transtemporal approach can provide excellent exposure of the intrapetrous internal carotid artery within the skull base to allow dissection or vascular manipulation. The consistent anatomy of the carotid canal can be important when the extracranial field has been distorted by disease processes, and this procedure can be helpful in providing anatomic orientation. This technique should be considered another important option in the armamentarium of the skull base surgeon for managing the internal carotid artery. 

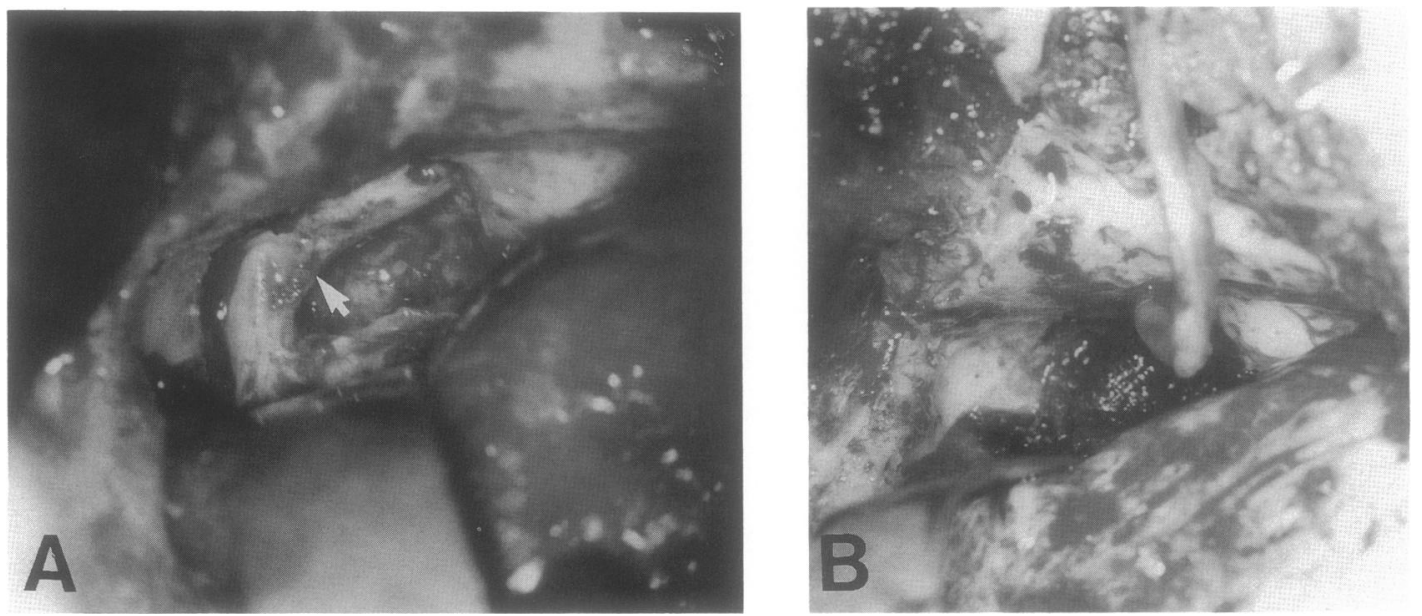

Figure 5. A: Exposure of the intrapetrous segment of the internal carotid artery for the patient in case 2. The diseased segment of the artery lies proximal to the fibrous band around the vessel (arrow). B: End-to-end anastomosis of a saphenous vein graft to the healthy distal horizontal segment of the internal carotid artery.

\section{REFERENCES}

1. Andrews JC, Valavanis A, Fisch U: Management of the internal carotid artery in surgery of the skull base. Laryngoscope 99 : 1224-1229, 1989

2. Konno A, Togawa $\mathrm{K}$, Iizuka $\mathrm{K}$ : Analysis of factors affecting complications of carotid ligation. Ann Otol Rhinol Laryngol 90 : 222-226, 1981

3. Glasscock ME: Middle fossa approach to the temporal bone: An otologic frontier. Arch Otolaryngol 90:41-53, 1969

4. Leonetti JP, Smith PG, Linthicum FH: The petrous carotid artery: Anatomic relationships in skull base surgery. Otolaryngol Head Neck Surg 102:3-12, 1990

5. Paullus WS, Pait TG, Rhoton AL: Microsurgical exposure of the petrous portion of the carotid artery. J Neurosurg 47:713-726, 1977

6. Hakuba A, Tanaka K, Suzuki T, Nishimura S: A combined orbitozygomatic infratemporal epidural and subdural approach for lesions involving the entire cavernous sinus. $\mathrm{J}$ Neurosurg 71 : 699-704, 1989

7. Sekhar LN, Schramm VL, Jones NF, Yonas H, Horton J, Latchaw $\mathrm{RE}$, Curtin $\mathrm{H}$ : Operative exposure and management of the petrous and upper cervical internal carotid artery. Neurosurgery 19:967-982, 1986

8. Hakuba A, Nishimura S, Jang BJ: A combined retroauricular and preauricular transpetrosal-transtentorial approach to clivus meningiomas. Surg Neurol 30:108-116, 1988

9. Fisch U, Oldring DJ, Senning A: Surgical therapy of internal carotid artery lesions of the skull base and temporal bone. Otolaryngol Head Neck Surg 88:548-554, 1980

10. Fisch U: Carotid lesions at the skull base. In Brackmann DE (ed): Neurological Surgery of the Ear and Skull Base. New York: Raven Press, 1982

11. Sekhar LN, Estonillo R: Transtemporal approach to the skull base: An anatomical study. Neurosurgery 19:799-808, 1986

12. Fisch U, Mattox D: Microsurgery of the Skull Base. New York: Thieme Medical Publishers, 1988

\section{REVIEWER'S COMMENTS}

The report by Andrews and associates is of value in reminding us of the intracranial exposure, provided by the middle fossa approach, to the petrous carotid artery. This is a given, known to most neurotologic skull base surgeons. What may be significant in the article is the modification of the incision in the temporalis muscle. This is similar to the "paddle"-like flap described by Pitanguay. We have found this additionally useful in: (1) repairs of tegmen defects, and (2) preservation of temporalis muscle for late facial reanimation procedures.

We agree with Andrews, et al that this type of wide field exposure is important for control of hemorrhage and have found it useful in these instances.

Richard J. Wiet, M.D., F.A.C.S. 